\title{
THE GAUSS REALIZABILITY PROBLEM ${ }^{1}$
}

MORRIS L. MARX

1. Introduction. Suppose $A=\left\{a_{1}, a_{2}, \cdots, a_{2 n}\right\}$ is a set of points in $(0,1)$ with $a_{i-1}<a_{i}, 2 \leqq i \leqq 2 n$. Let $*$ denote an involution of $A$ with no fixed points. Gauss [1] considered the question of when there exists a $C^{\prime}$ mapping $f$ from $[0,1]$ into $R^{2}$ such that $f(x)=f\left(x^{\prime}\right)$ if and only if $x=a_{i}, x^{\prime}=a_{i}^{*}$ for some $i, 1 \leqq i \leqq 2 n$, or $x=0, x^{\prime}=1$. It is further required that the derivative $f^{\prime}$ never vanish and that the vectors $f^{\prime}\left(a_{i}\right)$ and $f^{\prime}\left(a_{i}^{*}\right)$ be linearly independent, $1 \leqq i \leqq 2 n$. (Such a mapping is called normal; see [5, p. 1084].) If such an $f$ exists, then the pair $(A, *)$ is said to be realizable and $f$ is called a realization. Nagy [4] gave a proof of a necessary condition conjectured by Gauss; Titus [5] found a stronger necessary condition. In [6] Treybig gives necessary conditions that are also sufficient. The purpose of this paper is to give somewhat simpler necessary and sufficient conditions for realizability. These conditions are obtained from an easy modification of Lefschetz' proof [2] of Mac Lane's condition for the planarity of a finite graph [3].

2. Mac Lane's Theorem. For completeness, we give a version of Lefschetz' proof mentioned above.

By a graph $G$ we shall mean a one-dimensional simplicial complex. The one-dimensional simplexes will also be called edges. The geometrical complex associated with $G$ will be denoted $|G|$. The graph $G$ is called connected if the topological space $|G|$ is connected; $G$ is said to be separable if $|G|$ has a cut point. If there is a homeomorphism mapping $|G|$ into $R^{2}$ (or equivalently, $S^{2}$ ), then $G$ is called planar. We may assume $G$ is nonseparable since every finite graph decomposes into a finite number of nonseparable subgraphs and $G$ is planar if and only if each of these subgraphs is [7]. Let $Z_{1}(G)$ denote the module of one-cycles of $G$ over the integers mod 2. A one-cycle $z$ is said to be simple if it represents a Jordan curve.

TheOREM 1 (Mac Lane). The nonseparable finite graph $G$ is planar if and only if there exist simple cycles $z_{0}, z_{1}, \cdots, z_{R}$ in $Z_{1}(G)$ such that

(1) Each edge of $G$ belongs to exactly two of the $z_{j}$.

(2) The collection $\left\{z_{1}, \cdots, z_{R}\right\}$ is a basis for $Z_{1}(G)$.

(3) $z_{0}=\sum_{i=1}^{R} z_{i}$.

Presented to the Society, January 26, 1969; received by the editors August 5, 1968.

1 This work was supported by the University Research Council of Vanderbilt University. 
Proof. Suppose $G$ is planar and let $h$ be a homeomorphism of $|G|$ into $S^{2}$. The cycles corresponding to the boundaries of the components of $S^{2}-h(|G|)$ have the desired properties.

Now suppose we have $z_{0}, z_{1}, \cdots, z_{R}$ satisfying (1), (2), and (3). Construct a two-dimensional simplicial complex $L$ as follows. Let $x_{1}, \cdots, x_{n}, y_{0}, y_{1}, \cdots, y_{R}$ be the vertices of $L$, where $x_{1}, \cdots, x_{n}$ are the vertices of $G$. Take as two-dimensional simplexes all sets of the form $e_{j} \cup\left\{y_{i}\right\}$, where $e_{j}$ is an edge occurring in $z_{i}, 0 \leqq i \leqq R$. The one-dimensional simplexes of $L$ will be all one-dimensional faces of the above and all the one-simplexes of $G$. We shall show that $|L|$ is a two-dimensional manifold. Note that $|L|$ arises from $|G|$ by attaching a disk along its boundary to the simple closed curve $\left|z_{i}\right|$, $0 \leqq i \leqq R$. Since each edge $e$ lies on exactly two of the $z_{i},|L|$ is locally Euclidean at every point of the open geometrical simplex $\langle e\rangle$. We show that $|L|$ is locally Euclidean at vertex points.

Because of (1), we can find edges $e_{1}^{\prime}, e_{2}^{\prime}, \cdots, e_{8}^{\prime}$ at $x_{1}$ such that $e_{i}^{\prime}$ and $e_{i+1}^{\prime}$ belong to the simple cycle $z_{i}^{\prime}, 1 \leqq i \leqq s-1$, and $e_{1}^{\prime}$ and $e_{s}^{\prime}$ belong to the cycle $z_{s}^{\prime}$. If we show that there is no other edge containing $x_{1}$, then we have that $|L|$ is locally Euclidean at $x_{1}$. A similar proof shows that $|L|$ is locally Euclidean at $x_{k}, 2 \leqq k \leqq n$, and thus, we know that $|L|$ is a manifold. Suppose that $f$ is some other edge containing $x_{1}$. Since $G$ is nonseparable, there is some simple cycle $c$ containing $e_{1}^{\prime}$ and $f$, but no other edge at $x_{1}$. By (2), $c=\sum_{i \in H} z_{i}$ for some index set $H$. We know that $z_{1}^{\prime}$ or $z_{s}^{\prime}$ is present in the sum for $c$; without loss of generality, let us say that $z_{1}^{\prime}$ is present. We claim that every $z_{i}^{\prime}, 1 \leqq i \leqq s$, must be present. If not, let $h>1$ be the smallest index such that $z_{h}^{\prime}$ is not in the expression for $c$. Then $z_{h-1}^{\prime}$ is present and so $e_{h}^{\prime}$ is an edge for $c$; this contradicts the assumption that $c$ contains no edge at $x_{1}$ other than $e_{1}^{\prime}$ and $f$. Hence, we have that $z_{1}^{\prime}$ and $z_{s}^{\prime}$ are both present, but this implies that $e_{1}^{\prime}$ is present twice and cancels out. This is a contradiction.

The Euler characteristic of $|L|$ is $\alpha_{0}-\alpha_{1}+\alpha_{2}$ where $\alpha_{i}$ is the number of $i$-dimensional simplexes of $L, i=0,1,2$. The first Betti number of both $|L|$ and $|G|$ is $R$. It is well known that $\alpha_{0}-\alpha_{1}=1-R$. We have then that the Euler characteristic of $|L|$ is

$$
\left(\alpha_{0}+\alpha_{1}\right)+\alpha_{2}=(1-R)+R+1=2 .
$$

Thus, $|L|$ is a sphere and $G$ is planar.

3. Realizability. Suppose we are given a pair $(A, *)$ as in the introduction. Define a graph $G$ with respect to the pair as follows. Let the vertices of $G$ be the points $x_{1}, x_{2}, \cdots, x_{n}$. Let $g$ be a mapping 
from $A$ onto the set of vertices such that $g(t)=g\left(t^{\prime}\right)$ if and only if $t=a_{i}, t^{\prime}=a_{i}^{*}$ for some $i$. The graph $G$ has edges $e_{1}, \cdots, e_{2 n}$, where $e_{j}$ joins $g\left(a_{j}\right)$ and $g\left(a_{j+1}\right), 1 \leqq j \leqq 2 n-1$, and $e_{2 n}$ joins $g\left(a_{1}\right)$ and $g\left(a_{2 n}\right)$. We say the pair $(A, *)$ has property $Q\left[6\right.$, p. 225] if for each $a_{k}<a_{k}^{*}$, we have some $a_{j}$ such that $a_{k}<a_{j}<a_{k}^{*}<a_{j}^{*}$ or $a_{j}<a_{k}<a_{j}^{*}<a_{k}^{*}$. If $(A, *)$ does not have property $Q$, then $A$ decomposes into subsets $A_{1}, A_{2}, \cdots, A_{q}$ such that $(A, *)$ is realizable if and only if $\left(A_{i}, *\right)$ is realizable for $1 \leqq i \leqq q$. Note that the graph $G$ will be nonseparable if $(A, *)$ has property $Q$. It can happen that $G$ as defined has multiple edges. This causes no difficulty; as usual, break each multiple edge into two edges joined at a vertex. If $(A, *)$ has property $Q$, then $G$ cannot have loops.

DEFINITION. A cycle $z$ in $Z_{1}(G)$ will be called admissible if whenever $e_{j}$ is in $z, e_{j+1}$ is not, $1 \leqq j \leqq 2 n-1$ and if $e_{2 n}$ is in $z, e_{1}$ is not.

Theorem 2. Suppose $(A, *)$ is a pair with property $Q$ and $G$ is its associated graph. Then $(A, *)$ is realizable if and only if there exist simple admissible cycles $z_{0}, z_{1}, \cdots, z_{R}$ in $Z_{1}(G)$ which satisfy (1), (2), and (3) of Theorem 1.

Proof. If $G$ arises from a realizable $(A, *)$ with realization $f$, then the cycles corresponding to the boundaries of the components of $S^{2}-f([0,1])$ are admissible and satisfy (1), (2), (3).

Now suppose we have admissible cycles $z_{0}, z_{1}, \cdots, z_{R}$ which satisfy (1), (2), (3). As in the previous proof, we construct the complex $L$ such that $|L|$ is a sphere. The function $g$ we now consider as a mapping of $A$ into $|L|$. We extend $g$ to a mapping $f$ of $[0,1]$ onto $|G| \subset|L|$ in such a way that $f$ is a homeomorphism on the intervals $\left(0, a_{1}\right),\left(a_{2 n}, 1\right)$, and $\left(a_{j}, a_{j+1}\right), 1 \leqq j \leqq 2 n-1$; also, $f(0)=f(1)$. There is, of course, no differentiable structure on $|L|$, but the important aspect of normality of the realization is the following: Let $\beta, \gamma \subset[0,1]$ be the intervals containing $a_{j}$ and $a_{j}^{*}$, respectively, such that $\beta$ and $\gamma$ contain no other $a_{k}, a_{k} \neq a_{j}, a_{j}^{*}$. Then $f(\beta)$ and $f(\gamma)$ must not be tangent at $f\left(a_{j}\right)$, but must cross. That this happens is an immediate consequence of the way $L$ was constructed and the fact that the cycles used in constructing $L$ are admissible.

\section{REFERENCES}

1. C. F. Gauss, Werke, Teubner, Leipzig, 1900, pp. 272, 282-286.

2. S. Lefschetz, Planar graphs and related topics, Proc. Nat. Acad. Sci. U.S.A. 54 (1965), 1763-1765.

3. S. Mac Lane, A combinatorial condition for planar graphs, Fund. Math. 28 (1937), 22-32. 
4. J. V. Sz. Nagy, Über ein topologisches Problem von Gauss, Math. Z. 26 (1927), 579-592.

5. C. J. Titus, $A$ theory of normal curves and some applications, Pacific J. Math. 10 (1960), 1083-1096.

6. L. B. Treybig, A characterization of the double point structure of the projection of a polygonal knot in regular position, Trans. Amer. Math. Soc. 130 (1968), 223-247.

7. H. Whitney, Non-separable and planar graphs, Trans. Amer. Math. Soc. 34 (1932), 339-362.

VANDERBILT UNIVERSITY 